\title{
The Resistance Measurement Method of the Conducting Textiles
}

\author{
Lefayet Sultan Lipol \\ Fareast International University, Dhaka \\ Mayedul Islam \\ Bangladesh Standard Testing Institute. Dhaka \\ Nazrima Sultana \\ Fareast International University, Dhaka
}

doi: 10.19044/esj.2016.v12n27p242 URL:http://dx.doi.org/10.19044/esj.2016.v12n27p242

\begin{abstract}
The report describes the technique to measure the resistance of some novel conducting threads. All of the measurements were performed in the EMC lab at SP Technical Research Institute of Sweden, Boras. The conducting threads were received from Ms. Azadeh Soroudi of the University of Boras. The conducting additive used for the threads was Carbon Black. The resistance was measured using two different methods: The two-terminal measurement method and the four-terminal measurement method. A result from the comparison of the two methods was that the two terminal measurement method provided more information than the fourterminal method. There seemed to be no advantage to use the four terminal method at all, for the resistance range studied.
\end{abstract}

Keywords: Novel conducting threads, Picoammeter, Electrometer, Fixer, Multimeter, Two Terminal Resistance Measurement Method, Four Terminal Resistance measurement method

\section{Introduction}

A conductive textile fiber is a material in fiber form (long and extremely thin) that has a conductivity in the interval $10^{-6}-10^{6} \mathrm{~S} / \mathrm{cm}$. Synthetic fibers normally have a very low conductivity of less than $10^{-7}$ $\mathrm{S} / \mathrm{cm}$. Other requirements are that the fiber should be suitable for textile applications and for the different production steps in the textile industry. If the end product needs to be washable, the conducting fiber also needs to be washable. Another important aspect of the conducting fiber is that it shall not be harmful to the environment. Known application areas for conducting fibers are for example antistatic, ESD shielding, heating and power transfer. 
Other application areas for conducting fibers might be as sensors or to propagate signals.

Novel conducting threads are definitely different from the classical conducting threads and the electrical behavior is interesting and often something slightly strange.

\section{Test Method}

The resistance of the sample is determined at a number of test voltages value, using one or both of the different test methods, the two-point method and the four-point method.

\section{Scope}

The resistance measurement method can be used for the threads, bundles of threads and thin strips of fabrics.

\section{Test environment}

The test was performed in the ESD lab where the relative humidity was $(12 \pm 3) \%$ and the temperature was $(23 \pm 2)^{0} \mathrm{C}$.

\section{Two-terminal measurement method}

The resistance is calculated by dividing the measured voltage by the measured current. The current is the correct current through the sample, but measured voltage includes the voltage drop over the ampere meter as shown in figure 1, where $\mathrm{U}$ indicates the voltage source; $\mathrm{V}$ the voltmeter; and $\mathrm{A}$ the current meter. The test sample (conducting thread) is also indicated in the figure where the effective length for measurement was 9 centimeters and actual length of the thread was 48 centimeters (same as four point measurement).

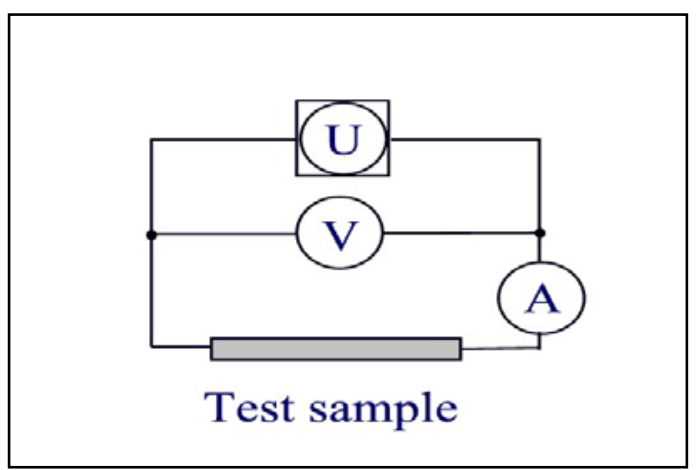

Figure-1: Two-terminal measurement method

The accuracy of the measurement is also limited by the voltage drop at the contact points between the electrodes and the sample. These errors can be eliminated by using a four-terminal method followed by a comparison of 
both method results. [Lars Fast, SP Technical Research Institute of Sweden, 2009]

\section{Four-terminal measurement method}

The resistance is calculated by dividing the measured voltage by the measured current. The voltage drop over the tested part of the sample is correct, but the measured current through the sample includes the current through the voltmeter. It is important that the resistance through the specified part of the sample is essentially lower than the resistance of the voltmeter and also that the contact resistance between the sample and the voltmeter is essentially lower than the resistance of the voltmeter. In the figure 2, $U$ indicates the voltage source and $\mathrm{V}$ the voltmeter and $\mathrm{A}$ indicates the current meter. For the two-point measurement method, two crocodile clips are used; one is positive (red) and another is negative (black) (Picoammeter). But for four-point measurement 4 crocodile clips are used: two for Picoammeter (red/black or +/-) and two for Electrometer (red/black or +/-). The distance of the Picoammeter crocodile clips was 18 centimeters for our measurement. All of the above distances were measured from the centre point of the fixtures ring. In case of the four-point measurement the voltage reading for the Picoammeter and Electrometer as well as the current of the Picoammeter were noted.

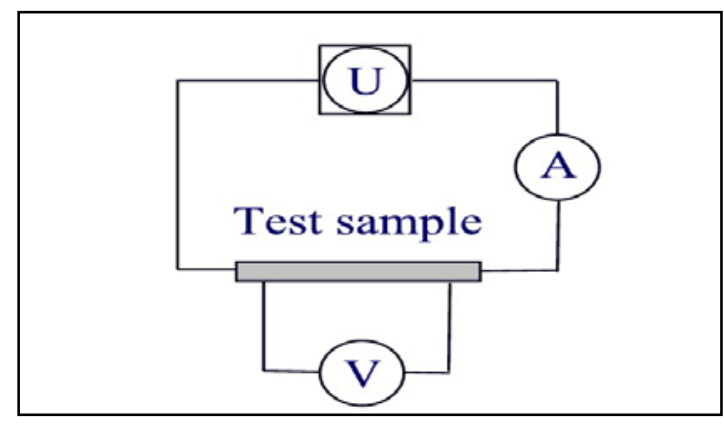

Figure-2: Four-terminal measurement method

The contact resistance between the sample and the electrodes can be of non-linear nature and the sample itself can also have non linear properties. Note that if the contact points of the current feed are the same as contact points for the voltage measurement, then the contact point is included in the resistance measurement. [Lars Fast, SP Technical Research Institute of Sweden, 2009]

\section{Conductive material}

There are three main materials that make the fiber conductive:

1. Metal

2. Carbon 
-Carbon black

-Graphite

-Carbon nanotube

3. Conductive polymer:

-Polyaniline (PANi)

- Poly (3, 4-ethylenedioxythiophene) (PEDOT)

- Polypyrrole (PPy)

The increase of conductivity can be obtained by additives to the bulk material, either homogenously distributed or for example as a surface coating. Metal is usually not infused but added as very thin conducting threads to the non-conducting threads, where the amount of metal thread can be adjusted to the needs of the manufacturer. [2, 3, 4]

\section{Experimental set-up}

The equipment used during our experiments is presented shortly-

\section{Picoammeter/Voltage source}

Brand name: Keithley, Range: up to $500 \mathrm{~V}, 2 \mathrm{~mA}$.

Function: By this machine, we can measure I (Amp), R $(\Omega)$, Voltage (V). In addition, we can supply electricity to the tested samples by this. This machine can measure the maximum voltage difference $500 \mathrm{~V}$ and maximum current $2 \mathrm{~mA}$. This machine is shown in figure 3 and it is situated third to the right.

\section{Electrometer}

Brand name: Keithley 617, Range: Up to $250 \mathrm{~V}, 2$ mA.

Function: It cannot supply electricity to the tested samples like Picoammeter. But we can measure I (Amp), R $(\Omega)$, U (Volt) by this instrument. This machine can measure the maximum voltage difference 250 $\mathrm{V}$ and maximum current $2 \mathrm{~mA}$. The machine is shown in figure 3 , second to the right.

\section{Multimeter}

Brand name: Fluke, True RMS Multimeter.

Function: By Multimeter we can measure current I (Amp), Voltage $\mathrm{U}$ (Volt), Resistance R $(\Omega)$ etc. Not shown in figure 3.

\section{Fixture}

This instrument was made in SP workshop. The fixture was made by silver metal. It is used to hold the tested threads and to connect the crocodile clips with the threads. With this fixture a $50 \mathrm{~g}$ dead weight is attached. We could not use the $50 \mathrm{~g}$ dead weight for our tested samples. The reason was 
that the threads could not bear this weight and extended on this weight and broke. So we used an $11.0 \mathrm{~g}$ crocodile clip for that. We used four brass rings to guide the conducting threads and the silver conducting paint was used to attach the conducting thread with the brass rings.

We used alcohol to remove the grease from the fixture when we received it from the SP Workshop. The reason is that grease can affect the resistance values for the experiment. We were ensured of removal of grease from the fixture by measuring the resistance of the silver made Fixture on its different places by Multimeter. The instrument is shown in the figure 3 first to the right.

\section{Silver conductive paint}

Brand name: Electrolube.

Function: To contact the thread with fixture. It should be shaken properly before it is used and we need to use a wood stick to use over fixture.

\section{Crocodile clips}

Function: To contact the electrical instrument with the thread. We could not use $50 \mathrm{gm}$ dead weight for our tested samples. The reason was that the threads could not bear this weight and broke. So we used a lighter weight crocodile clip for that.

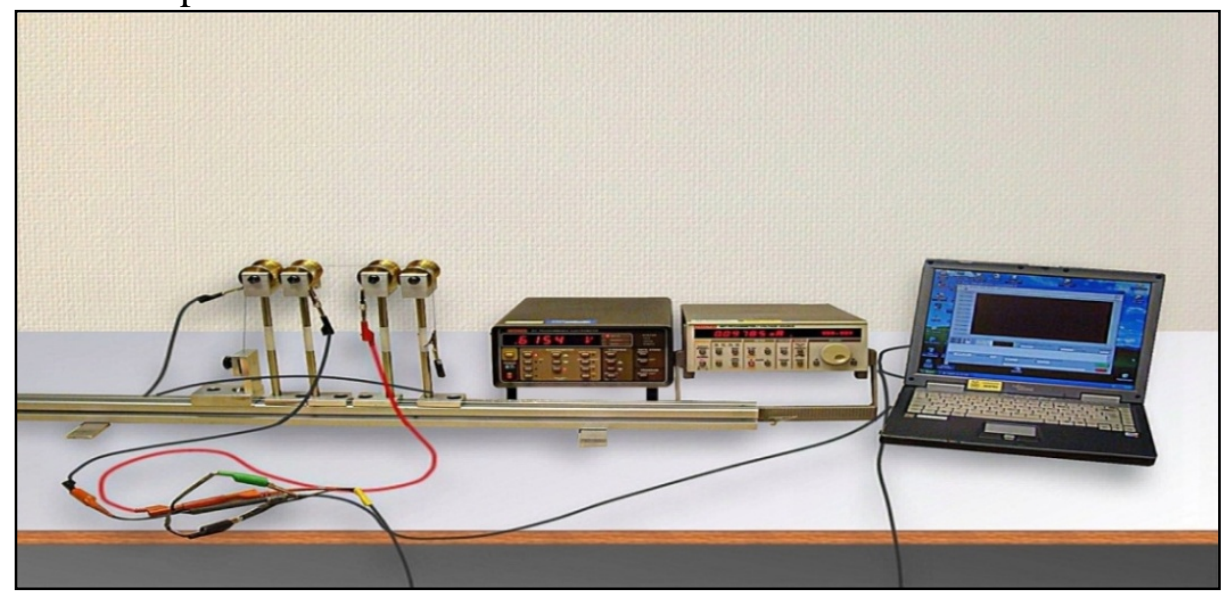

Figure-3: Experimental set-up to measure resistance of the conductive textiles [Lars Fast, SP Technical Research Institute of Sweden, 2009]

\section{Small preliminary experimental measurements}

Resistance measurement of the copper wire

In table 1 we present the resistance of a thin copper wire as a function of the applied voltage. The resistance is as expected, depending on the applied voltage. 


\begin{tabular}{|c|c|c|c|c|c|}
\hline $\mathrm{U}_{2}(\mathrm{~V})$ & $\mathrm{I}(\mathrm{A})$ & $\mathrm{R}_{2}(\mathrm{~W})$ & $\mathrm{U}_{4}(\mathrm{~V})$ & $\mathrm{R}_{4}(\Omega)$ & $2 * \mathrm{R}_{\mathrm{c}}(\mathrm{W})$ \\
\hline 0.006 & 0.039 & 0.154 & $*$ & $*$ & $*$ \\
\hline 0.031 & 0.256 & 0.121 & 0.005 & 0.020 & 0.102 \\
\hline 0.162 & 1.300 & 0.125 & 0.024 & 0.018 & 0.106 \\
\hline 0.255 & 2.290 & 0.111 & 0.044 & 0.019 & 0.092 \\
\hline 0.326 & 4.620 & 0.071 & 0.091 & 0.020 & 0.051 \\
\hline
\end{tabular}

Table-1: The resistance of a thin copper wire as function of applied voltage.

In the table 1, we measured a copper wire by two and four point measurement method for some specific voltages. The resistance values (R) are not as linear as for two points but are linear for four point measurement method. In addition, we have shown the difference of datas for a specific voltages for the above mentioned methods. It is observed that the difference decreases for higher voltages. As the contact resistances are not included in the four point measurement method (as they were in the two point measurement method), we can say the four point measurement method is preferable. [Lars Fast, SP Technical Research Institute of Sweden, 2009]

\section{Resistance measurement of a novel conducting thread}

Samples of Azadeh Soroudi

Table 2: The resistance is presented as function of the applied voltage

\begin{tabular}{|c|c|c|c|}
\hline Uin $(\mathrm{V})$ & $\mathrm{R}(\Omega)$ & Uin $(\mathrm{V})$ & $\mathrm{R}(\Omega)$ \\
\hline 0.001 & $*$ & 70 & $5.00 \mathrm{E}+05$ \\
\hline 0.002 & $*$ & 80 & $5.00 \mathrm{E}+05$ \\
\hline 0.005 & $*$ & 90 & $5.01 \mathrm{E}+05$ \\
\hline 0.01 & $8.33 \mathrm{E}+07$ & 100 & $5.04 \mathrm{E}+05$ \\
\hline 0.02 & $9.09 \mathrm{E}+07$ & 120 & $5.00 \mathrm{E}+05$ \\
\hline 0.05 & $1.06 \mathrm{E}+08$ & 140 & $5.19 \mathrm{E}+05$ \\
\hline 0.1 & $1.16 \mathrm{E}+08$ & 160 & $5.16 \mathrm{E}+05$ \\
\hline 0.2 & $5.00 \mathrm{E}+05$ & 180 & $5.14 \mathrm{E}+05$ \\
\hline 0.5 & $5.00 \mathrm{E}+05$ & 200 & $5.26 \mathrm{E}+05$ \\
\hline 1 & $4.98 \mathrm{E}+05$ & 220 & $5.37 \mathrm{E}+05$ \\
\hline 2 & $4.96 \mathrm{E}+05$ & 240 & $5.45 \mathrm{E}+05$ \\
\hline 5 & $4.96 \mathrm{E}+05$ & 260 & $5.42 \mathrm{E}+05$ \\
\hline 10 & $4.96 \mathrm{E}+05$ & 280 & $5.38 \mathrm{E}+05$ \\
\hline 20 & $4.97 \mathrm{E}+05$ & 300 & $£$ \\
\hline 30 & $4.97 \mathrm{E}+05$ & & \\
\hline 40 & $4.97 \mathrm{E}+05$ & & \\
\hline 50 & $4.97 \mathrm{E}+05$ & & \\
\hline 60 & $4.98 \mathrm{E}+05$ & & \\
\hline
\end{tabular}

N.B: $*=$ Not measurable, $£=$ Burnt. 
From the table 1 and 2 we can say that conducting threads are essentially five magnitudes less conductive than the copper wire and that they show a very strong non-linear behavior for voltages less than $0.2 \mathrm{~V}$. [Lars Fast, SP Technical Research Institute of Sweden, 2009]

\section{Discussion}

We used the four-terminal and the two-terminal methods for measuring the resistance of the samples. During the measurement with fourterminal measurement methods, we used four contacts points of which two contacts for the Picoammeter and two contacts for the Electrometer. On the other hand, for the two-terminal measurement methods, we used only Picoammeter and two contact points. In case of two-terminal measurement method, the effective length for measuring the resistance was 9 centimeters (the distance of the crocodile clips of Picoammeter). On the other hand, in the case of the four-terminal measurement, the effective length for measurement was 18 centimeters (the distance of the crocodile clips of Picoammeter). But in that case, the distance of the crocodile clips of the Electrometer was 9 centimeters. Both techniques gave similar result for higher voltages. In the example of copper wire (Table-1), we showed the advantage of four-terminal measurements. However, in the case of samples (Table-2), we saw no such advantage as the conducting threads showed very high resistances. When we applied low voltage, the threads showed very high resistance and unusual behavior. The resistance showed consistency for higher applied voltages, but it could not bear a voltage difference above $500 \mathrm{~V}$ in most cases. As a result, the threads were burnt.

\section{Conclusion}

We used silver conductive paints for contacting the samples with the fixture properly but we are unsure about the actual conductivity of this silver paint and its effect on the threads. In addition, we used four brass rings for guiding the conducting materials, but its contribution to the resistance measurement is unknown to us. We hope to work further on these problems in the next part of this project.

\section{References:}

Thesis Supervisor: Lars Fast PhD, SP Technical Research Institute of Sweden (on behalf of the University of Boras, Sweden) \& Research Assistant: Lefayet Sultan Lipol (2009), M. Sc in Textile Engineering program of the University of Boras-Thesis work named Resistance Measurement of the Novel Conducting Threads at SP Technical Research Institute of Sweden at Boras, EMC lab. 
http://hyperphysics.phy-astr.gsu.edu/Hbase/electric/elepow.html, date: 3006-2016

http://en.wikepedia.org/wiki/Electrical_resistance, date: 15-06-2016

http://www.kespec.freeuk.com/resistan.htm, date: 10-06-2016 